\title{
Effect studies of Uyghur sand therapy on the hemodynamics of the knee-joint arteries
}

\author{
Rongchang $\mathrm{Fu}^{*}$, Dilinaer Mahemut, Rexiati Tiyipujiang, Kuwahan Aihemaiti and Nuerya \\ Ainiwaierjiang \\ Department of Mechanical Engineering, Xinjiang University, Xinjiang 830047, China
}

\begin{abstract}
This paper studies the effect of Uyghur sand therapy on dynamics of arterial flow of knee joints via experiments and numerical simulations. Experiments have been carried out on 30 volunteers, with their diameter and flow rate of arteries of knee joints measured before and after Uyghur sand therapy. It has been found that Uyghur sand therapy will increase the inner diameter of knee arteries and speed up the blood flow. Experimental results show that Uyghur sand therapy can help relieve obstacles in local blood flow. By choosing one volunteer for CT scanning, three-dimensional reconstruction of kneejoint arteries via MIMICS software is achieved. Calculation model is the established with numerical calculations performed by ANSYS software. According to the calculations, the blood flow of the knee arteries speeds up and the uniform distribution of velocity enlarges after Uyghur sand therapy, which further confirms the experimental results. Besides, the research also suggests that Uyghur sand therapy has stronger effect on blood flow of knee-joint arteries than the inner diameter.
\end{abstract}

Keywords: Uyghur sand therapy, hemodynamics, finite element analysis, velocity of blood flow

\section{Introduction}

As an important part of Uyghur medicine, Uyghur sand therapy has drawn an increasing amount of interest due to its obvious curative effect on all kinds of arthritis, including rheumatism, chronic lumbocrural pain, sciatica, angiitis, chronic appendagitis, dermatosis and etc. Besides, its curative effect on some diseases in nerve systems and blood pressure are also at mild or moderate levels [1].

Uyghur sand therapy can be described as burying the diseased parts into the hot sand and cure diseases via synthesis of heat, magnetism and strength. Due to multiple influencing factors and disciplines involved, up till now, theoretical researches only achieve macroscopical qualitative analysis level and no quantitative analysis has been reported.

Previous studies show significant curative effect of Uyghur sand therapy on Osteoarthritis (OA) [2]. $\mathrm{OA}$ is one of the most common chronic diseases and it is a clinical syndrome with a major pathological feature in a set of knee articular cartilage [3]. Up till now, many studies show that there exists a certain relation between the change of the hemodynamic indices and the occurrence, development, treatment and prevention of OA [4]. Meanwhile, hemodynamic indices are closely related to blood

\footnotetext{
${ }^{*}$ Corresponding author: Rongchang Fu, Department of Mechanical Engineering, Xinjiang University, No. 1230, Yanan Road, Urumqi, Xinjiang 830047, China. Tel.: 008613699357563; Fax: 008609918592308; E-mail: changrong2010@sina.com.
} 
flow [5,6]. As a result, this paper studies the effect of Uyghur sand therapy on dynamics of arterial flow of knee joints via experiments and numerical simulations, which aims at further disclosure of the hemorheological mechanism of Uyghur sand therapy. The achievement in this study can be best seen as the development of natural resources and the progress in economy and tourism in Turpan area.

\section{Experimental material and method}

\subsection{Experimental subject}

15 male and 15 female volunteers are selected as the experimental subjects. Male subjects have an average height of $172 \mathrm{~cm}$ (ranging from 168 to $175 \mathrm{~cm}$ ) and weight of $75 \mathrm{~kg}$, while female subjects have an average height of $165 \mathrm{~cm}$ (ranging from 158 to $170 \mathrm{~cm}$ ) and weight of $55 \mathrm{~kg}$.

\subsection{Experimental method}

Before applying the Uyghur sand therapy, AG Color Doppler B-ultrasound is used to measure blood vessel diameter and blood flow velocity of knee-joint arteries. The Uyghur sand therapy is applied by fastening treatment cushion on knee joints, followed by electrifying and heating for 20 mins. After-
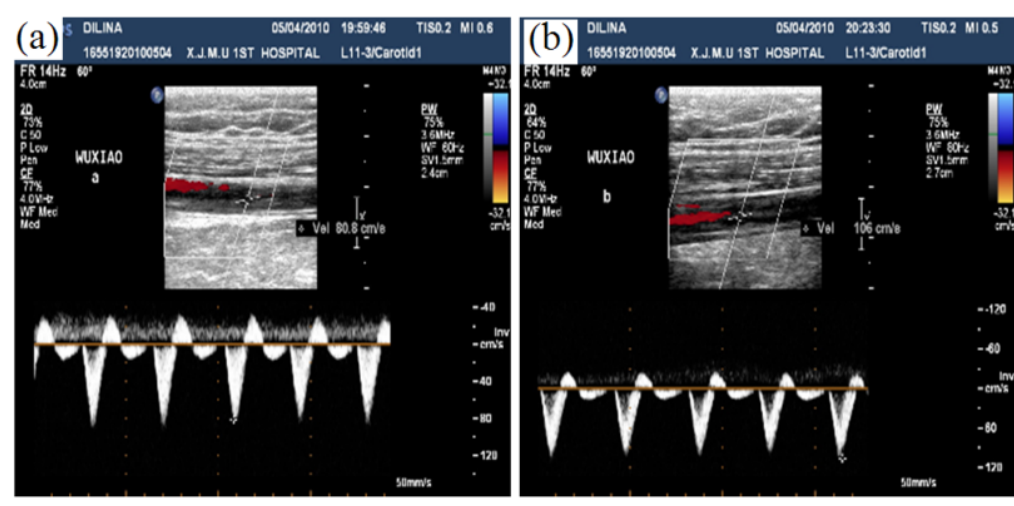

Fig. 1. Blood flow velocity of knee-joint arteries (a) before and (b) after Uyghur sand therapy.
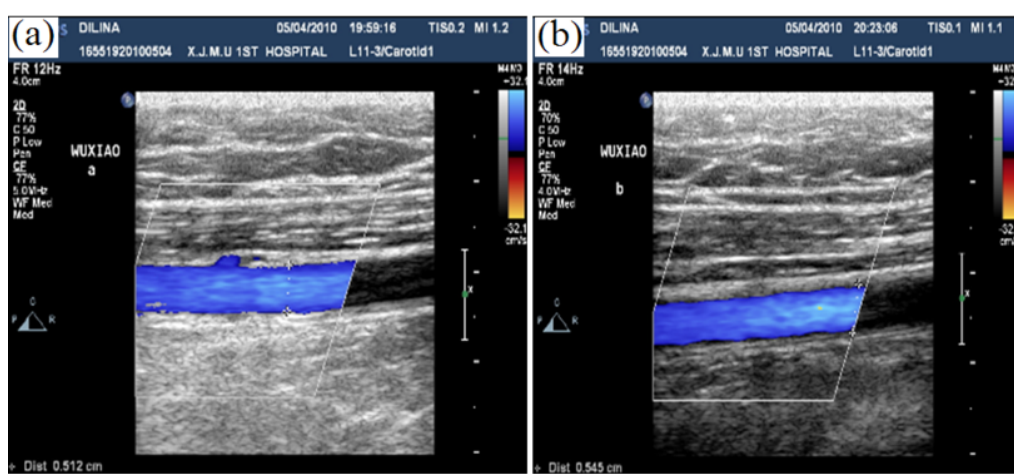

Fig. 2. Inner diameter of knee-joint arteries (a) before and (b) after Uyghur sand therapy. 
wards, blood vessel diameter and blood flow velocity of knee-joint arteries are measured again using AG Color Doppler B-ultrasound. These steps are repeated for 15 days before the entire experiment is completed. Figure 1 shows the comparison of blood flow velocity of knee-joint arteries before and after applying the therapy, while Figure 2 shows the comparison of inner diameter of knee-joint arteries before and after applying the therapy.

\subsection{Analysis of experimental result}

Test methods vary according to types of study design and purpose of statistical inference. According to the difference of inner diameter and blood flow velocity of the knee-joint arteries before and after Uyghur sand therapy, this paper concludes difference in effect of different levels of experimental factors on experimental indices as mean test of the significance of difference, namely paired sample analysis of mean value. In this paper, paired sample of data processing function in Excel is used to conduct the sample analysis. The analysis results can be found in Tables 1 and 2.

As we can see in Tables 1 and 2, after applying Uyghur sand therapy, both blood vessel diameter and blood flow velocity experience statistically significant increase (enlarged diameter and increased

Table 1

Blood vessel diameter of knee-joint arteries before and after applying Uyghur sand therapy

\begin{tabular}{lll}
\hline & $\begin{array}{l}\text { Blood vessel diameter } \\
\text { Uyghur sand therapy }\end{array}$ & $\begin{array}{l}\text { before } \\
\text { Blood vessel diameter after Uyghur } \\
\text { sand therapy }\end{array}$ \\
\hline Average & 0.562333333 & 0.602111111 \\
Variance & 0.00326625 & 0.002046361 \\
Observed value & 9 & 9 \\
$\begin{array}{l}\text { Poisson coefficient of } \\
\text { correlation }\end{array}$ & 0.524046741 & \\
Supposed mean deviation & 0 & \\
df & 8 & \\
t Stat & -2.33899516 & \\
P(T<=t) single tail & 0.023745062 & \\
t double tail criticality & 1.859548033 & \\
$\mathrm{P}(\mathrm{T}<=\mathrm{t})$ double tail & 0.047490124 & \\
$\mathrm{t}$ double tail criticality & 2.306004133 & \\
\hline
\end{tabular}

Table 2

Blood flow velocity of knee-joint arteries before and after applying Uyghur sand therapy

\begin{tabular}{lll}
\hline & $\begin{array}{l}\text { Blood flow velocity before Uy- } \\
\text { ghur sand therapy }\end{array}$ & $\begin{array}{l}\text { Blood flow velocity after Uyghur } \\
\text { sand therapy }\end{array}$ \\
\hline Average & 84.575 & 99.1375 \\
Variance & 224.7021429 & 277.4398214 \\
Observed value & 8 & 8 \\
$\begin{array}{l}\text { Poisson coefficient } \quad \text { of } \\
\text { correlation }\end{array}$ & 0.582904707 & \\
Supposed mean deviation & 0 & \\
df & 7 & \\
t Stat & -2.835163758 & \\
$\mathrm{P}(\mathrm{T}<=\mathrm{t})$ single tail & 0.012609399 & \\
$\mathrm{t}$ single tail criticality & 1.894578604 & \\
$\mathrm{P}(\mathrm{T}<=\mathrm{t})$ double tail & 0.025218798 & \\
$\mathrm{t}$ double tail criticality & 2.364624251 & \\
\hline
\end{tabular}


velocity). Specifically, the therapy has stronger effect on blood flow velocity than vessel diameter.

Heating is an important feature of Uyghur sand therapy. It warms up human tissues, which would help to increase oxygen saturation, accelerate heart rate, dilate blood vessels. Besides, as heat could increase blood flow velocity of knee-joint arteries of lower limbs of the cured part, notable improvements can be seen in blood circulation, which assists diseases treatment.

\section{Analog calculation}

\subsection{Theoretical model}

Blood flow of arteries is an important topic in biofluid mechanics with many problems in the dynamics of pulsating flow unsolved. Some major problems include nonlinearity of blood flow, nonlinear elasticity of vascular wall (or nonlinearity viscoelasticity), blood and vascular pulsation coupling, finite deformation of artery blood vessel and etc. The synthesis of these features increases the complexity of blood flow research. In particular, the geometrical shape of artery blood vessel and the coupled motion of blood vessel and blood fluid make the problem remain unsolved, a problem unable to be solved with effect.

For simplicity, in this paper, we assume the blood vessels can be regarded as circular section rigid pipes. The validity of this assumption is backed up by the observation that in the main human arteries, blood vessel diameters only fluctuate $5-10 \%$. As for the vessel walls, it is generally treated as rigid pipes. Even if arteries with pathological changes are involved, their vessel walls are usually thickened and hardened and with reduced vessel wall motions, which makes it reasonable to treat them as rigid pipes.

The blood fluid is assumed to be incompressible Newtonian fluid [7]. Combining with the assumption of rigid pipe, Navier-Stokes equation (N-S equation) can be used to describe the blood fluid motion as follows with Eq. (1) as the continuity equation and Eq. (2) the N-S equation [8,9].

$$
\left.\begin{array}{l}
\frac{\partial u}{\partial x}+\frac{\partial v}{\partial y}+\frac{\partial w}{\partial z}=0 \\
\frac{\partial u}{\partial t}+u \frac{\partial u}{\partial x}+v \frac{\partial u}{\partial y}+w \frac{\partial u}{\partial z}-\mu\left(\frac{\partial^{2} u}{\partial x^{2}}+\frac{\partial^{2} u}{\partial y^{2}}+\frac{\partial^{2} u}{\partial z^{2}}\right)+\frac{1}{\rho} \frac{\partial p}{\partial x}=0 \\
\frac{\partial v}{\partial t}+u \frac{\partial v}{\partial x}+v \frac{\partial v}{\partial y}+w \frac{\partial v}{\partial z}-\mu\left(\frac{\partial^{2} v}{\partial x^{2}}+\frac{\partial^{2} v}{\partial y^{2}}+\frac{\partial^{2} v}{\partial z^{2}}\right)+\frac{1}{\rho} \frac{\partial p}{\partial y}=0 \\
\frac{\partial w}{\partial t}+u \frac{\partial w}{\partial x}+v \frac{\partial w}{\partial y}+w \frac{\partial w}{\partial z}-\mu\left(\frac{\partial^{2} w}{\partial x^{2}}+\frac{\partial^{2} w}{\partial y^{2}}+\frac{\partial^{2} w}{\partial z^{2}}\right)+\frac{1}{\rho} \frac{\partial p}{\partial z}=0
\end{array}\right\}
$$

where $u, v, w$ are the blood flow speed in $x, y, z$ direction in Cartesian coordinates, respectively. $p$ is the pressure intensity, $p$ is the blood density and $u$ is the dynamic viscosity.

Boundary conditions, depending on the specific circumstances of the flow field, will provide velocity and pressure values at the boundaries. As for static vessel walls, the speed must satisfy zero slip condition, namely, $u=v=w=0$. 
After establishing the mathematical model, hemodynamic numerical simulations are employed to solve control equations of the abovementioned flow with certain initial and boundary conditions provided.

\subsection{Calculation model}

To obtain the geometrical shape of true knee-joint artery blood vessels, we carry out a threedimensional reconstruction via CT scanning and MIMICS software. MIMICS is a 3D image producting and editing processor. Various scanned data (CT or MRI) can be loaded into MIMICS to establish a 3D model. After editing, universal CAD (computer aided design), FEA (finite element analysis) and RP (rapid prototyping) graphs can be generated, which converts and manages large-scale data in PC. Figure 3 shows a graph generated from CT data loaded into MIMICS and Figure 4 shows the 3D modeling of knee-joint arteries.

\subsection{Calculation conditions}

Solving the blood flow control equation involves solving pressure intensity and three velocity components, which require initial conditions and boundary conditions. In this paper, we use definite analysis for calculation and investigation of physiological pulsating flow in knee-joint artery vessels. As for mesh generation, finite element uses four node tetrahedron element and free meshing.

Figures 1 and 2 show the blood flow velocity and inner diameter of knee-joint arteries of one experimental subject with detailed parameter values as follows. The vessel diameter (D) before and after applying the therapy are 0.512 and $0.545 \mathrm{~cm}$, respectively. As for the blood flow velocity peak values $\left(\mathrm{V}_{\max }\right)$ they are 80.8 and $106 \mathrm{~cm} / \mathrm{s}$ before and after the therapy. The vessel length $(l)$ is $3 \mathrm{~cm}$, the pulse cycle (T) is $0.6 \mathrm{~s}$, the blood density $(\rho)$ is $1.056 \mathrm{~g} / \mathrm{cm}^{3}$, and the dynamic viscosity $(\mu)$ is $0.003 \mathrm{pa} \cdot \mathrm{s}$.

Recall the equation for Reynold number [10],

$$
R_{e}=\frac{\rho V_{\max } D}{\mu}
$$

We have the Reynolds number of 1456 and 2033 before and after the therapy. As both numbers are below 2320, we could treat the blood flow as laminar.

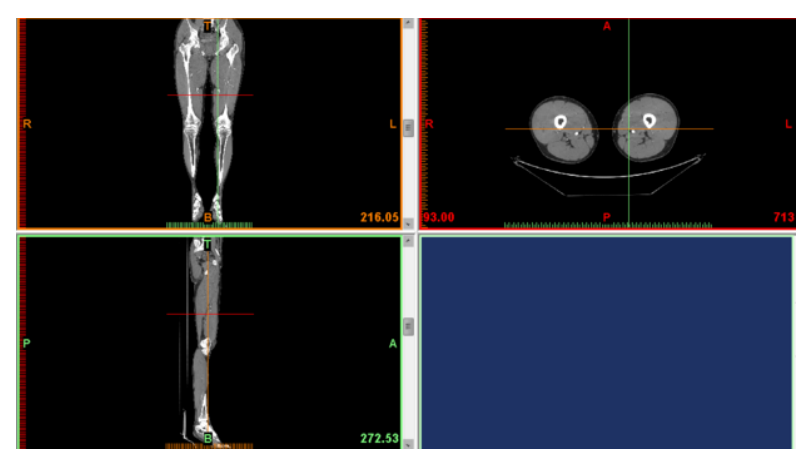

Fig. 3. Graph derived from inputting CT data in MIMICS.

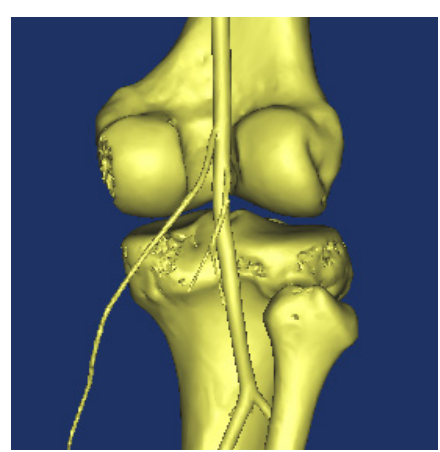

Fig. 4. 3D modeling of knee-joint arteries. 
Entrance velocity before the treatment can be described as $v_{x}=0, v_{y}=0, v_{z}=80.8\left|\sin \left(\frac{t \pi}{T}\right)\right|$

And the entrance velocity after the treatment can be described as $v_{x}^{\prime}=0, v_{y}^{\prime}=0, v_{z}^{\prime}=106\left|\sin \left(\frac{t \pi}{T}\right)\right|$ with an exit pressure $P$ of zero.

As for the computing control, steady algorithm is applied by assuming laminar blood flow and incompressible Newtonian fluid. Stable results can be obtained after iterative calculations.

\subsection{Calculation result and analysis}

Figure 5 shows the axial velocity profile before and after the Uyghur sand therapy. The velocity peaks at the axis and reduces along the radius towards the wall. Besides, the maximum speed increases after applying therapy.

Figure 6 shows the axial pressure profile before and after the Uyghur sand therapy. Obvious pressure gradient can be observed along the flow direction before and after the Uyghur sand therapy, while the gradient after applying therapy is more evident.

Figure 7 shows shearing stress distribution along radial direction before and after Uyghur sand therapy. The shearing stress peaks at the wall and reduces along the radius towards the axis. Besides, the shearing stress value becomes larger after the Uyghur sand therapy.

Numerical simulations are performed upon blood flow of knee-joint arteries using ANSYS software with the following conclusions obtained.

- Blood flows in the knee-joint arteries are all laminar flows. The flow velocity varies with the slowest near the vessel wall and the highest near the vascular shaft. Besides, areas with higher velocity expand after Uyghur sand therapy, which indicates improved blood flow.

- Obvious pressure gradients can be seen in blood flow in artery blood vessels both before and after Uyghur sand therapy and the gradient increases after therapy, indicating a faster blood flow.

- The shearing strength peaks at the vessel wall, and reduces in the direction towards the vessel shaft. Besides, the shearing strength increases after the Uyghur sand therapy. Therefore, in the whole cardiac cycle, the possibility of thrombus deposition reduces. The blood could then flow more smoothly, giving an impetus to blood circulation.
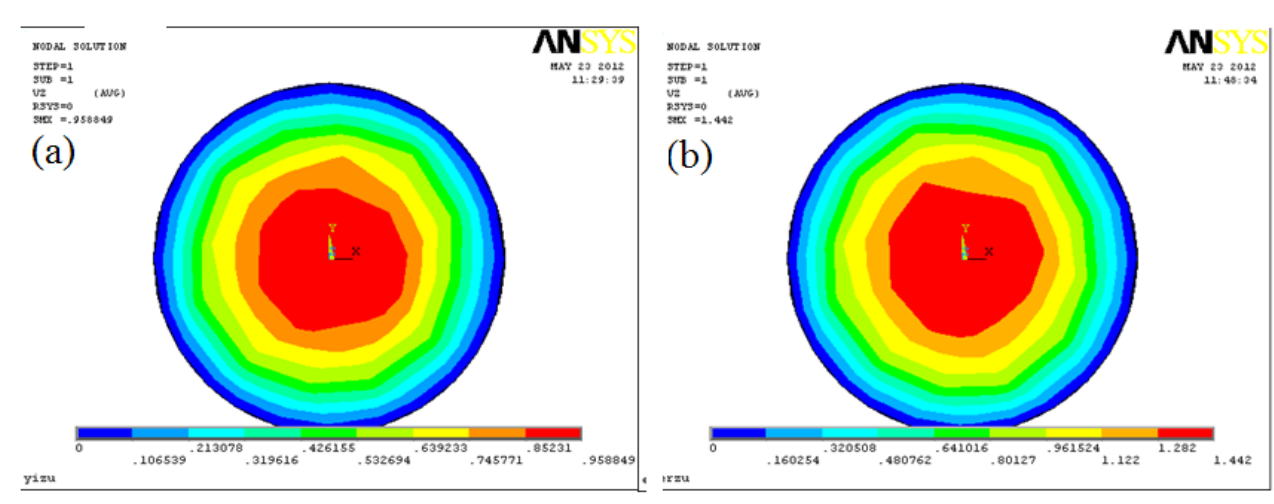

Fig. 5. Axial velocity profile (a) before and (b) after the Uyghur sand therapy. 


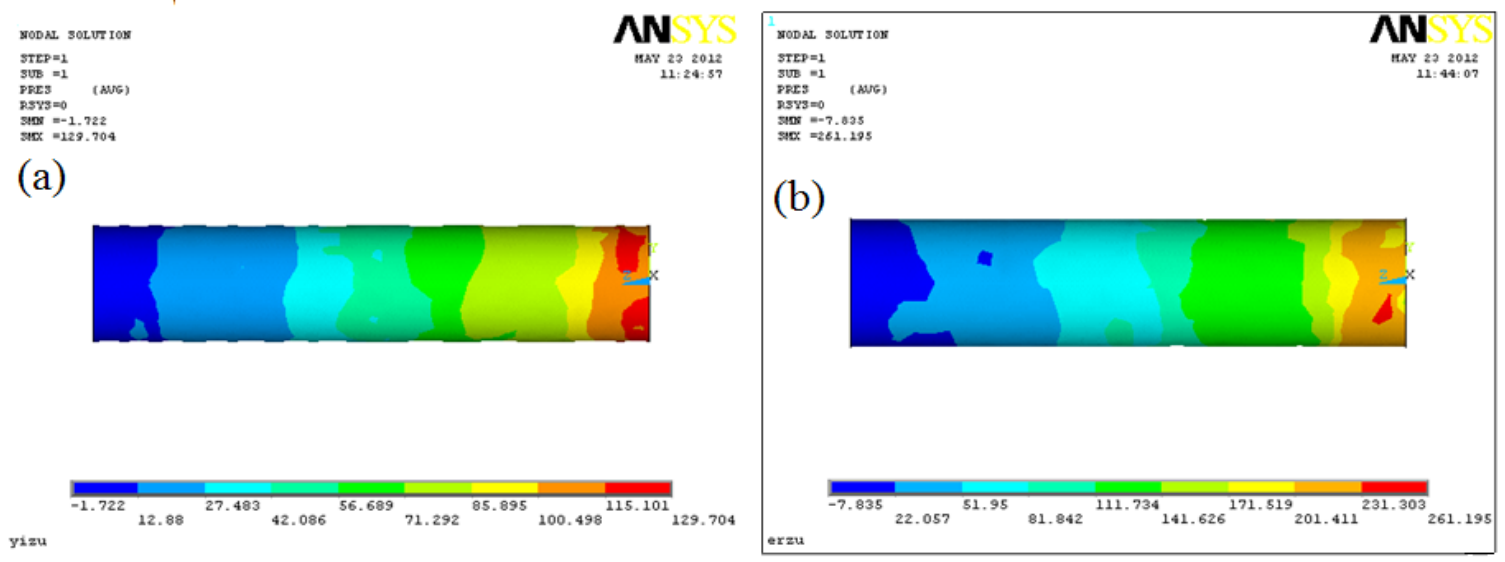

Fig. 6. Pressure distribution in vessel shaft direction (a) before and (b) after Uyghur sand therapy.
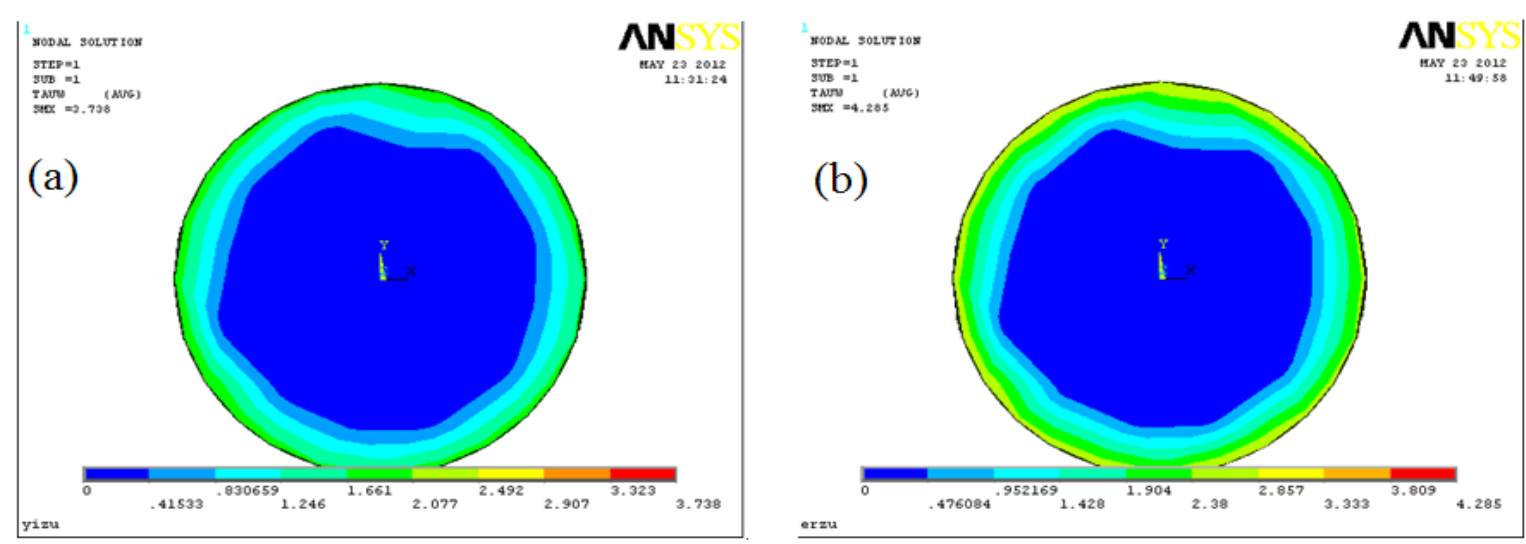

Fig. 7. Shearing stress distribution along radial direction (a) before and (b) after Uyghur sand therapy.

\section{Conclusion}

This paper studies the effect of Uyghur sand therapy on dynamics of arterial flow of knee joints using experiments and numerical simulations. As confirmed by both simulations and experiments, Uyghur sand therapy can expand the inner diameter of knee arteries, speed up the blood flow and help to enlarge the uniform distribution of velocity. Besides, Uyghur sand therapy has stronger effect on blood flow of knee-joint arteries than the inner diameter. Therefore, Uyghur sand therapy can improve blood circulation which helps with disease treatment and experiments.

\section{Acknowledgement}

This paper is supported by the Natural Science Foundation of the Xinjiang Uygur Autonomous Region (2014211A005), China. 


\section{References}

[1] D. Mahemuti, H. Wupuer and R.F., Niyazi, Biomechanics and uyghur sand therapy, Journal of Medicine \& Pharmacy of Chinese Minorities 6 (2000), 24-25.

[2] N. Aishan, Research of uyghur sand therapy on treatment rheumatism in xinjiang tulufan, Journal of Medicine \& Pharmacy of Chinese Minorities 8 (2002), 21-22.

[3] L. Galois, S. Etienne, C. Henrionnet, J. Scala-Bertola, L. Grossin, D. Mainard, P. Gillet and A. Pinzano, Ambivalent properties of hyaluronate and hylan during post-traumatic OA in the rat knee, Bio-Medical Materials and Engineering 22 (2012), 235-242.

[4] L. Yang, C.Q. He, W. Xie, R.-B. Yan and J. Chen, Hemorheological change in patients with knee osteoarthritis: Multiple factor variance analysis and cross section observation, Clinical Rehabilitative Tissue Engineering Research 13 (2009), 2053-2056.

[5] A. Toth, J. Papp, M. Rabai, P. Kenyeres, Zs. Marton, G. Kesmarky, I. Juricskay, H.J. Meiselman and K. Toth, The role of hemorheological factors in cardiovascular medicine, Clinical Hemorheology and Microcirculation 56 (2014), 197204.

[6] R.F. Sofi, A.A. Liotta, S. Fedi, C. Macchi, G. Pratesi, R. Pulli, C. Pratesi, R. Abbate and L. Mannini, Alterations of haemorheological parameters in patients with peripheral arterial disease, Clinical Hemorheology and Microcirculation 55 (2013), 271-276.

[7] Z.Z. Wang, A.Q. Sun, Y.B. Fan and X.Y. Deng, comparative study of Newtonian and non-Newtonian simulations of drug transport in a model drug-eluting stent, Biorheology 49 (2012), 249-259.

[8] G.-M. Gie and C.-Y. Jung, Vorticity layers of the 2D navier-stokes equations with a slip type boundary condition, Asymptotic Analysis 84 (2013), 17-33.

[9] N. Antonova, X. Dong, P. Tosheva, E. Kaliviotis and I. Velcheva, Numerical analysis of 3D blood flow and common carotid artery hemodynamics in the carotid artery bifurcation with stenos, Clinical Hemorheology and Microcirculation 57 (2014), 159-173.

[10] J.-H. Moh, Y.I. Cho, D.J. Cho, D. Kim and R.K. Banerjee, influence of non-newtonian viscosity of blood on microvascular impairment, Clinical Hemorheology and Microcirculation 57 (2014), 111-118. 